\title{
Gold Nanodisks Plasmonic Array for Hydrogen Sensing at Low Temperature
}

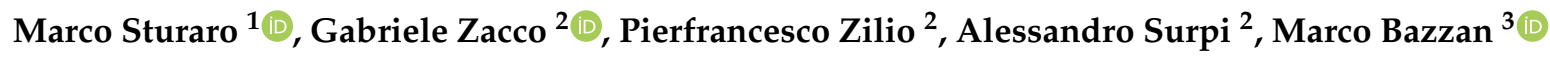 \\ and Alessandro Martucci ${ }^{1, *(D)}$ \\ 1 Dipartimento di Ingegneria Industriale, Università di Padova, 35131 Padova, Italy; sturarom@gmail.com \\ 2 Veneto Nanotech, 35100 Padova, Italy; gabriele.zacco@al-lighting.com (G.Z.); \\ pierfrancesco.zilio@gmail.com (P.Z.); asurpi@bo.ismn.cnr.it (A.S.) \\ 3 Dipartimento di Fisica e Astronomia, Università di Padova, 35131 Padova, Italy; marco.bazzan@unipd.it \\ * Correspondence: alex.martucci@unipd.it
}

Received: 21 December 2018; Accepted: 31 January 2019; Published: 5 February 2019

\begin{abstract}
We present a novel plasmonic hydrogen sensor consisting of an array of gold nanodisks produced by lithography. The size, height, and spacing of the disks were optimized using finite element simulation to generate a sharp localized surface plasmon resonance peak in the near-infrared wavelength region. The reported results show the possibility of developing an optical gas sensors-based bare Au nanostructures operating at a low temperature.
\end{abstract}

Keywords: plasmonic; gold array; optical gas sensors; hydrogen sensors; nanoimprinting

\section{Introduction}

Gold is regarded as relatively inert to chemical interactions with gases and generally considered a poor catalyst. Instead, gold nanoclusters up to $5 \mathrm{~nm}$ of diameter show catalytic activity to $\mathrm{CO}$ and $\mathrm{H}_{2}$ oxidation [1,2], especially when supported by transition metal oxides [3]. The reactivity of small $\mathrm{Au}$ nanocrystals (NCs) is largely attributed to the presence of low-coordinated Au atoms, abundant in nanometer-sized nanoparticles (NPs) [4]. Furthermore, Density Functional Theory (DFT) simulations confirm that the crucial condition for $\mathrm{H}_{2}$ interaction with gold is the existence of low-coordinated atoms, independently if on the surface of Au NCs or at extended line defects [5]. In the latter case, it is possible to observe significant catalytic activity in larger gold structures. For example, Fujita et al. [6] studied CO oxidation activity in nanoporous gold, where the feature characteristic lengths were larger than $30 \mathrm{~nm}$ but with peculiar defective surfaces comparable to 3-5 nm Au NCs curvature. Recently, bare gold nanostructures have been exploited to obtain hydrogen sensors at low temperature. The proposed mechanism involves hot electron-induced hydrogen molecule dissociation and subsequent metastable hydride formation $[7,8]$.

In this paper, we study the interactions of $\mathrm{H}_{2}$ with a metamaterial consisting of bare gold nanodisks (Au NDs) arrays on silica substrate. The aim was to verify the possibility of optically detecting hydrogen at low temperature using only gold as the sensitive material without any particular excitations and exploiting the shift in the localized surface plasmon resonance (LSPR) of the gold array.

\section{Materials and Methods}

\subsection{Fabrication Method}

A master was realized on silicon substrate by means of Lloyd Interferential Lithography (LIL) and inductively coupled plasma (ICP) etching. In the second phase, the master on silicon was replicated 
numerous times on quartz substrates. The last phase of the process comprised metallization of chromium and gold by electron beam evaporation and a subsequent lift-off.

The master mold was produced by an interferential lithography system in a "Lloyd's Mirror" configuration. In this configuration, the interference pattern is created by the superposition of a portion of a laser beam directly reaching the sample and another portion of the beam reflected by a mirror placed at $90^{\circ}$ to the sample. The light source used was a $50 \mathrm{~mW} \mathrm{HeCd}$ laser emitting a single mode at $\lambda=325 \mathrm{~nm}$ and $30 \mathrm{~cm}$ coherence length. The laser beam was directed on a spatial filter to eliminate spurious frequencies and then continued free to propagate, achieving a distance of $2 \mathrm{~m}$ and reaching the Lloyd interferometer.

Bottom Anti-Reflective Coating (BARC) was deposited onto silicon by spin coating at $3000 \mathrm{rpm}$ for $30 \mathrm{~s}$ and annealed on a hot plate at $175^{\circ} \mathrm{C}$ for $1 \mathrm{~min}$. Photoresist was a SPR 220 diluted in propylene glycol methyl ether acetate (PGMEA), deposited by spin coating at $5000 \mathrm{rpm}$ for $30 \mathrm{~s}$ and placed on a hot plate for $90 \mathrm{~s}$.

Sample obtained after lithography constituted NDs of photoresist on BARC/silicon. Subsequently, a transfer process was performed using ICP etching. The master obtained, shown in Figure S2 of Supplementary Materials (SM), was then used as a mold for nanoimprinting. Quartz slides $\left(6 \mathrm{~cm}^{2}\right)$ were cleaned in ultrasonic bath and washed for several cycles with water, soap, and isopropyl alcohol. Next, mr-I 7010 R thermoplastic polymer-Microresist (MRI), was deposited on quartz by spin coating (1500 rpm for $30 \mathrm{~s}$ and annealed at $100{ }^{\circ} \mathrm{C}$ for $1 \mathrm{~min}$ ). Quartz slide and mold were then joined at a pressure of $100 \mathrm{bar}$ and $100^{\circ} \mathrm{C}$ for $10 \mathrm{~min}$. Finally, chromium and gold evaporation by electron beam was performed.

\subsection{Characterizations}

The morphology of the NDs array was investigated with a xT Nova NanoLab Scanning Electron Microscopy (SEM).

X-ray diffraction $(\mathrm{XRD})$ using the $\mathrm{CuK}_{\alpha}(\lambda=1.54056 \AA)$ radiation was performed using a Philips MRD diffractometer, equipped with a parabolic multilayer mirror for primary beam conditioning and an Anton-Paar sample chamber which allowed investigation of the structural characteristics of the samples in a controlled atmosphere and temperature. The diffracted beam was measured as a function of the scattering angle by a proportional Xe counter coupled to a Parallel Plate Collimator with an angular acceptance of $0.01^{\circ}$.

Transmittance at normal incidence and ellipsometry quantities $\Psi$ and $\Delta$ were measured using a J.A. Woollam V-VASE Spectroscopic Ellipsometer in vertical configuration at a fixed angle of $70^{\circ}$ in the wavelength range 300-1500 $\mathrm{nm}$. Refractive index $\mathrm{n}$ and extinction coefficient $\mathrm{k}$ have been evaluated from $\Psi, \Delta$, and transmittance data using the WVASE32 ellipsometry data analysis software fitting the experimental data with Cauchy dispersion and Gaussian oscillators. A cell that allows heating and introduction of gases (nitrogen and a mixture of $5 \mathrm{vol} \%$ of $\mathrm{H}_{2}$ in $\mathrm{Ar}$ at $0.4 \mathrm{~L} / \mathrm{min}$ ) was used to observe changes in sample optical constants in the presence of hydrogen.

Optical gas sensing tests were performed via optical absorption measurements over the wavelength range $300 \mathrm{~nm}<\lambda<2000 \mathrm{~nm}$ using a Harrick gas flow cell coupled with a Jasco V-570 spectrophotometer. NDs arrays deposited on $\mathrm{SiO}_{2}$ substrates were exposed at different operative temperatures (from 30 to $300{ }^{\circ} \mathrm{C}$ ) to $1 \% \mathrm{v} / \mathrm{v} \mathrm{H}_{2}$ balanced in dry air, at a flow rate of $0.4 \mathrm{~L} / \mathrm{min}$. All tested samples were stabilized at the operative temperature for one hour in air before gas sensing measurements. Sensor sensitivity was assessed using response intensity (RI) defined as $\mathrm{RI}=\left|1-\mathrm{Abs}_{\mathrm{Gas}} / \mathrm{Abs}_{\mathrm{Air}}\right|$. To analyze the dynamic behavior of the sensor, we used the response and recovery time calculated as the time needed to reach $90 \%$ of the total response and to recover $90 \%$ of the baseline. 


\section{Results and Discussion}

LSPR absorption peak can be effectively tuned by changing the aspect ratio of Au NDs and the periodicity of the array [9]. By using COMSOL Multiphysics, a commercial software which uses finite element method (FEM), the optical response of a single gold ND in air having glass as substrate was modelled, and its radius and height were optimized to obtain a strong and sharp LSPR peak. Subsequently, the procedure was repeated for NDs square arrays to optimize ND spacing. Simulations (see Figure S1 of SM) indicate that increasing the ND diameter leads to a red shift of the LSPR peak position [9], passing from $\lambda_{\text {LSPR }}=700 \mathrm{~nm}$ (visible range) for a ND with radius of $25 \mathrm{~nm}$ to $\lambda_{\text {LSPR }}=1100 \mathrm{~nm}$ (NIR) for a ND with radius of $150 \mathrm{~nm}$. ND thickness increment, for every simulated width, had the opposite effect on the LSPR peak position (i.e., increasing thickness induced a blue shift of the LSPR peak position); $5 \mathrm{~nm}$ thickness was chosen for subsequent simulations because the NDs have the sharpest LSPR in the visible/NIR range for that thickness. Similar absorption cross sections for NDs arrays with period length between 250 and $450 \mathrm{~nm}$ at $\lambda_{\text {LSPR }} \sim 1100 \mathrm{~nm}$ were obtained with successive simulations (Figure 1). On the basis of simulations, samples containing NDs with $75 \mathrm{~nm}$ radius and $5 \mathrm{~nm}$ thickness with a periodicity of $400 \mathrm{~nm}$ were fabricated by nanoimprinting from a master mold produced by interferential lithography (Figure 1c) [10]. Using this approach, a large number of samples can be produced from a single master while maintaining high resolution and high experimental reproducibility.

The samples studied for $\mathrm{H}_{2}$ sensing were arrays of gold NDs on quartz, each with a radius of $75 \mathrm{~nm}$ and height $5 \mathrm{~nm}$ with a period of $400 \mathrm{~nm}$. The absorption spectra is shown in Figure 2, in which the LSPR is centered at $\sim 1100 \mathrm{~nm}$, as predicted by simulations. SEM images of the sample are presented in Figure 2, showing well-defined, long-range order and quite regular NDs shapes.

The sensing behavior of $\mathrm{Au}$ NDs was investigated using $\mathrm{H}_{2}(1 \mathrm{vol} \%$ in air) as target gas and dry air as reference gas. The samples were stabilized in air at the operative temperature for 1 hour before gas sensing measurements. Stabilization is important because the temperature affects the size of the NDs and hence the position of the LSPR, as reported in Figures S3 and S4 of the Supplementary Materials. The shift of the LSPR peak observed in Figure S4 is consistent with the simulations, which predict a blue shift of the LSPR peak as the diameter of the Au ND decreases (Figure S3).

As seen in Figure 3, the LSPR peak shifts to lower wavelengths, passing from air to $\mathrm{H}_{2}$ at different operative temperature; this effect is reversible. The effect of $\mathrm{H}_{2}$ exposure can be better appreciated by plotting the difference of the absorbance and the wavelength (Optical Absorbance Change, $\mathrm{OAC}=\mathrm{Abs}_{\mathrm{H}_{2}}-\mathrm{Abs}_{\mathrm{Air}}$ ), as reported in Figure 4. The response is wavelength dependent and influenced by the operative temperature, even if a detectable signal also can be obtained at $30{ }^{\circ} \mathrm{C}$. Figure 4a shows that the OAC has a maximum at $~ 950 \mathrm{~nm}$ (for all operative temperatures) and a minimum (dependent on operative temperatures) in the 1100-1400 nm range, which can be used for dynamic tests. Figure $4 \mathrm{~b}, \mathrm{c}$ show the dynamic tests at $950 \mathrm{~nm}$ and for two operative temperatures $\left(100^{\circ} \mathrm{C}\right.$ and $150{ }^{\circ} \mathrm{C}$ ) for two air $/ \mathrm{H}_{2} /$ air cycles. The sensor exhibits better performance at higher operative temperatures in terms of response, recovery time, and response intensity (RI), which were estimated (using the data reported in Figure 4c) to be $100 \mathrm{~s}, 140 \mathrm{~s}$, and $0.1 \%$, respectively. At temperatures above $20{ }^{\circ} \mathrm{C}$, sensitivity declines and the LSPR peak tends to decrease in intensity as a result of the gradual change of the Au NDs dimension, as reported in Figures S3 and S4 of the supplementary material. These experiments demonstrate that our bare Au NDs array can detect hydrogen at relatively low temperature, a phenomenon observed before for bare Au only in cases of very small gold clusters [5] or involving hot electrons $[7,8]$.

The observed LSPR shift could be related to a variation of the refractive index. However, the shift due to refractive index variation (hydrogen refractive index is slightly lower than air) is negligible if compared with the shift observed experimentally. Moreover, ellipsometry measurements showed no variations due to gas exposure (see Figure S5 in Supplementary Materials). 


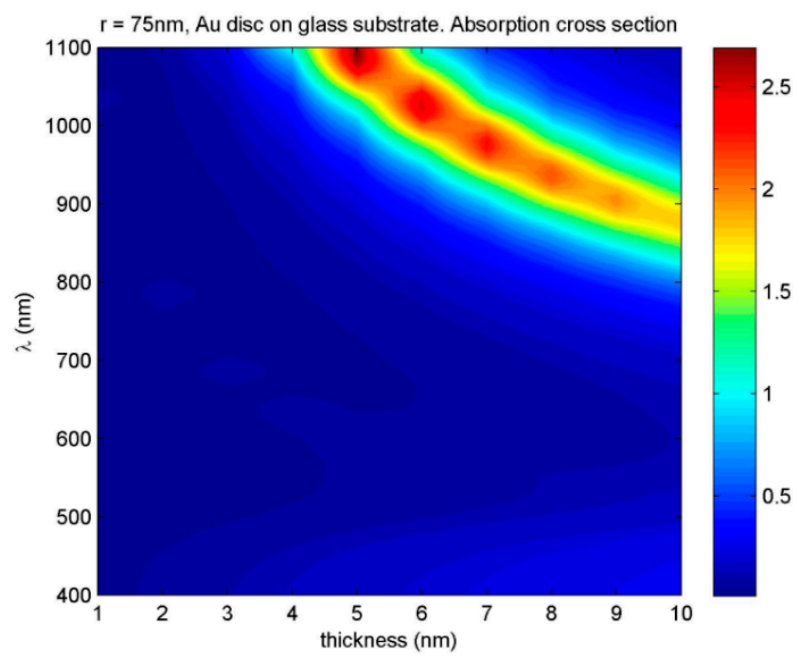

(a)

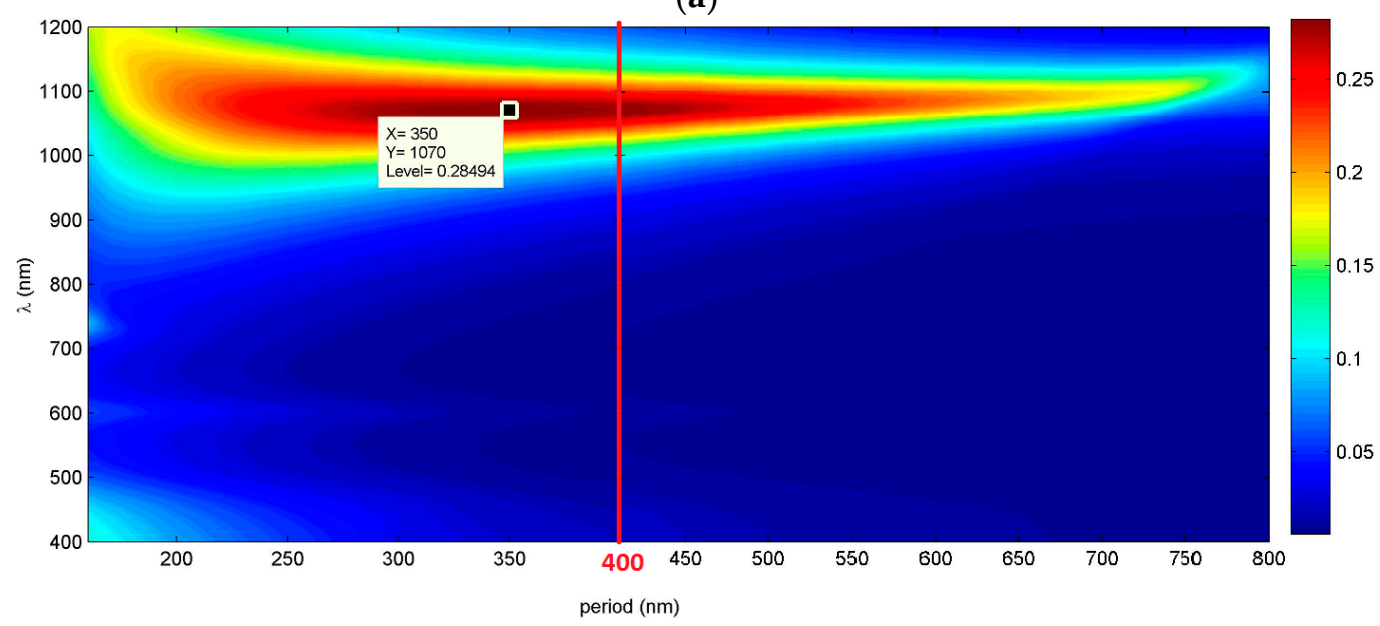

(b)

LIL Patterning ICP pattern Transfer Imprinting on MRI Lift Off

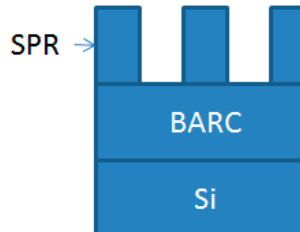

I fase: MASTER

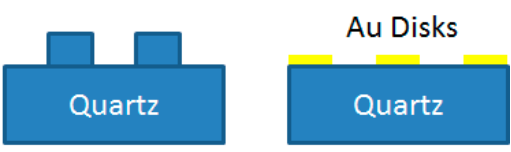

II fase: Repliche e liftoff

(c)

Figure 1. (a) Absorption cross-section of single Au ND as a function of wavelength and ND thickness for radius $=75 \mathrm{~nm}$; (b) Absorption cross section of Au NDs array as a function of wavelength and periodicity for radius $=75 \mathrm{~nm}$, thickness $=5 \mathrm{~nm}$; (c) Scheme of sample preparation method. 

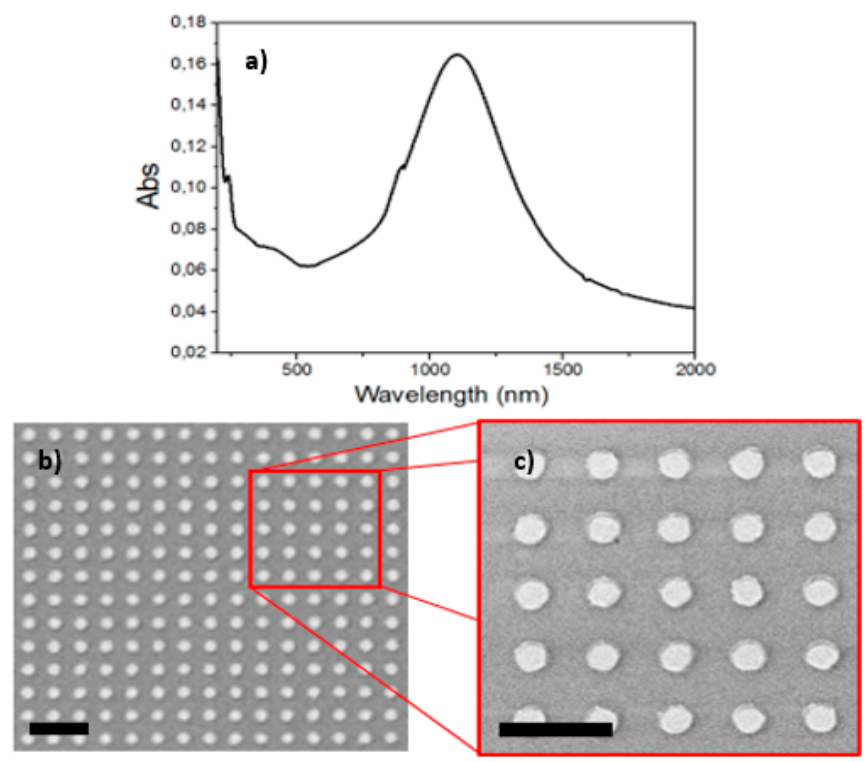

Figure 2. (a) Optical absorption spectrum in the 200-2000 nm range and (b,c) SEM images of Au NDs array on quartz with diameter of $150 \mathrm{~nm}$ and height of $5 \mathrm{~nm}$. Scale bars are $500 \mathrm{~nm}$.
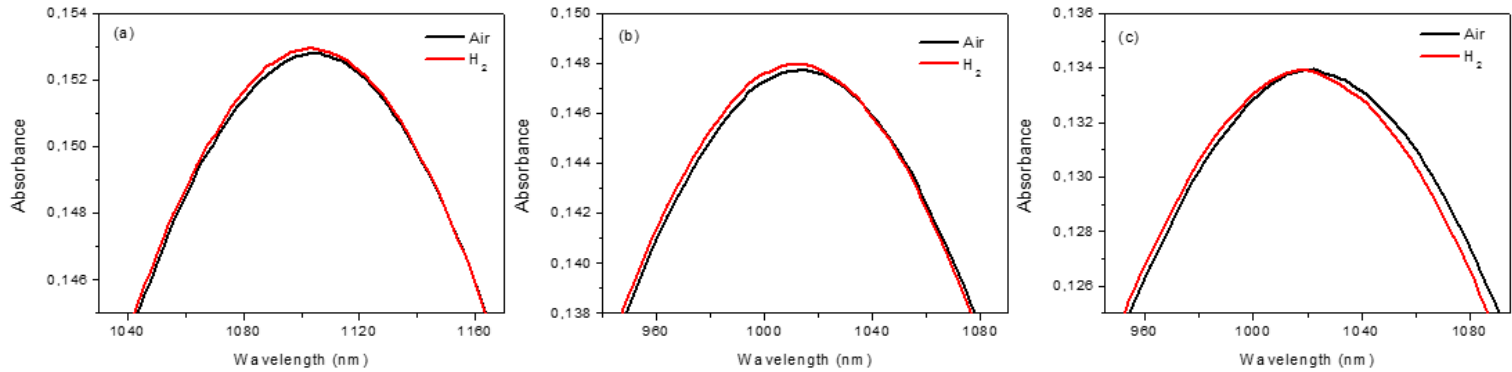

Figure 3. Optical absorption spectra for Au NDs at (a) $30{ }^{\circ} \mathrm{C},(\mathbf{b}) 100{ }^{\circ} \mathrm{C}$, and (c) $150{ }^{\circ} \mathrm{C}$ operative temperature in air (black line) and in $\mathrm{H}_{2}$ (red line).
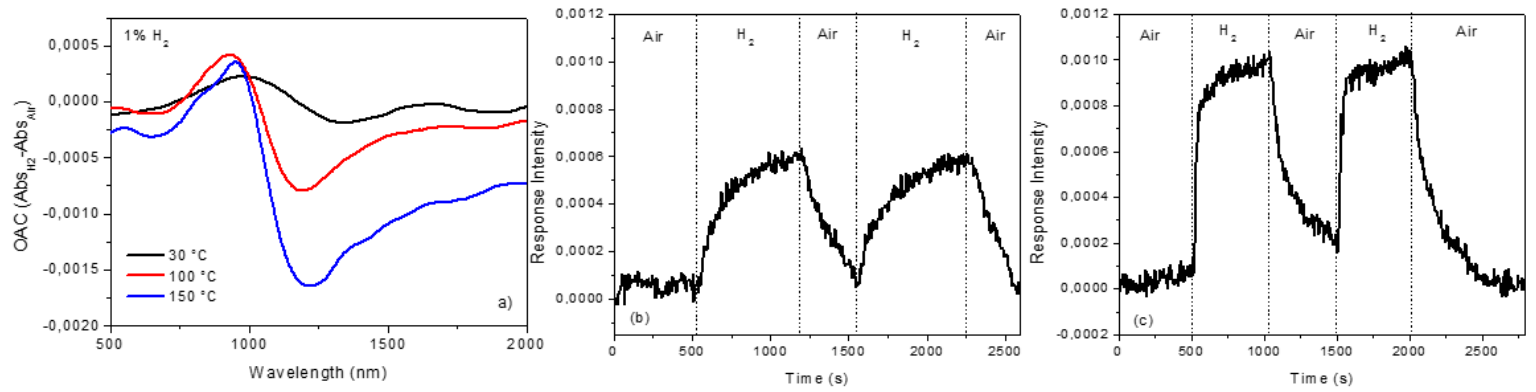

Figure 4. (a) Optical Absorbance Change, $\mathrm{OAC}=\mathrm{Abs}_{\mathrm{H}_{2}}-\mathrm{Abs}_{\mathrm{Air}}$, for $\mathrm{Au} \mathrm{NDs}$ at $30{ }^{\circ} \mathrm{C}, 100{ }^{\circ} \mathrm{C}$, and $150{ }^{\circ} \mathrm{C}$ operative temperature when exposed to $1 \% \mathrm{H}_{2}$ in air. Dynamic cycle air $/ \mathrm{H}_{2} /$ air at $950 \mathrm{~nm}$ and $100{ }^{\circ} \mathrm{C}(\mathbf{b})$ and $150{ }^{\circ} \mathrm{C}(\mathbf{c})$ operative temperature.

Another possible mechanism involves the formation of gold-hydrogen bonds [5], preferentially at the disks' edges, where gold atoms are low coordinated. We performed XRD measurements (see Figure S6 in SM) to investigate the influence of hydrogen interaction with the Au NDs, but the XRD diffraction peak of Au was not affected by the presence of hydrogen. This experiment does not exclude the hydrogen-gold bond formation because the hydrogen dissociation on gold would occur preferentially only on the surfaces edges, which is difficult to detect by standard XRD. Nevertheless, XRD measurements provided important information about Au crystallite size, calculated using the Scherrer equation. Au NDs are polycrystalline structures with a mean crystallite 
diameter of $6 \pm 0.5 \mathrm{~nm}$, the same order of magnitude of an Au cluster presenting activity to hydrogen dissociation $[1,2,5]$. However, direct hydrogen dissociation at low temperature has been demonstrated only for small $\mathrm{Au}$ clusters, supported on metal oxides such as $\mathrm{TiO}_{2}$ and $\mathrm{CeO}_{2}[11,12]$.

In our experiments, we did not use any type of light excitation source for exciting hot electrons as reported in $[7,8,13,14]$, obtaining a detectable shift also at $30^{\circ} \mathrm{C}$. Therefore, the formation of hot electrons seems to be not very likely to occur.

\section{Conclusions}

In summary, we present a new sensing platform for hydrogen on the basis of the enhanced sensing performance of bare gold resulting from the sharp plasmon resonance of a specifically designed nanodisks pattern fabricated by nanoimprinting. The Au NDs array showed a reversible response to hydrogen at low temperature with a response intensity of $0.1 \%$ and a response time of $100 \mathrm{~s}$ at an operative temperature of $150^{\circ} \mathrm{C}$. Although the mechanisms involved in the observed LSPR shift is not completely understood, we demonstrated the possibility to design a plasmonic structure made of bare gold with good sensitivity and reversibility for $\mathrm{H}_{2}$ sensing.

Supplementary Materials: The following are available online at http:/ / www.mdpi.com/1424-8220/19/3/647/s1, Figure S1: Absorption cross-section of a single Au ND as a function of wavelength and ND thickness for radius $\mathrm{r}=150 \mathrm{~nm}(\mathrm{a}), 75 \mathrm{~nm}$ (b), $50 \mathrm{~nm}$ (c), $25 \mathrm{~nm}$ (d), Figure S2, SEM images of masters used for samples with NDs diameters of $150 \mathrm{~nm}$ (left) and $50 \mathrm{~nm}$ (right). Scale bars are $1 \mu \mathrm{m}$ for both images, Figure S3, SEM images of the Au NDs array after (a) $30^{\circ} \mathrm{C}$, (b) $150{ }^{\circ} \mathrm{C}$, (c) $250^{\circ} \mathrm{C}$ stabilization in air for $1 \mathrm{~h}$. The mean diameter with the relative standard deviation are $166 \pm 10 \mathrm{~nm}, 152 \pm 9 \mathrm{~nm}, 143 \pm 11 \mathrm{~nm}$, respectively, Figure S4, Absorbance spectra of the $\mathrm{Au}$ NDs after stabilization at $30^{\circ} \mathrm{C}, 150^{\circ} \mathrm{C}$ and $250^{\circ} \mathrm{C}$ in air for $1 \mathrm{~h}$, Figure S5, Refractive index (n) and extinction coefficient $(\mathrm{k})$, respectively plotted in black and blue, for Au NDs in presence of inert atmosphere (nitrogen, solid curves) or hydrogen-rich atmosphere $\left(5 \mathrm{vol} \% \mathrm{H}_{2}\right.$ in Argon, dashed curves), Figure S6, XRD patterns for the (111) Bragg peak of Au NDs in presence of inert atmosphere (Argon, black curve) or hydrogen-rich atmosphere (5 vol\% $\mathrm{H}_{2}$ in Argon, red curve).

Author Contributions: Conceptualization, A.M. and A.S.; Methodology, A.M.; Validation, M.S. and A.S.; Investigation: XRD, M.B., Gas Sensing and Optical Characterizations, M.S. Fabrications and Simulations, A.S., G.Z., P.Z.; All authors contributed to interpretation of the results. Writing-Original Draft Preparation, M.S. with contributions from all authors; Writing-Review \& Editing, A.M.; Supervision, A.M.

Acknowledgments: We thank Veneto Nanotech for financial support.

Conflicts of Interest: The authors declare no conflict of interest.

\section{References}

1. Varganov, S.A.; Olson, R.M.; Gordon, M.S.; Mills, G.; Metiu, H. A study of the reactions of molecular hydrogen with small gold clusters. J. Chem. Phys. 2004, 120, 5169-5175. [CrossRef] [PubMed]

2. Haruta, M. When Gold Is Not Noble: Catalysis by Nanoparticles. Chem. Rec. 2003, 3, 75-87. [CrossRef] [PubMed]

3. della Gaspera, E.; Antonello, A.; Guglielmi, M.; Post, M.L.; Bello, V.; Mattei, G.; Romanato, F.; Martucci, A. Colloidal approach to Au-loaded $\mathrm{TiO}_{2}$ thin films with optimized optical sensing properties. J. Mater. Chem. 2011, 21, 4293-4300. [CrossRef]

4. Hvolbæk, B.; Janssens, T.V.W.; Clausen, B.S.; Falsig, H.; Christensen, C.H.; Nørskov, J.K. Catalytic activity of Au nanoparticles. Nano Today 2007, 2, 14-18.

5. Corma, A.; Boronat, M.; Gonzalez, S.; Illasb, F. On the activation of molecular hydrogen by gold: A theoretical approximation to the nature of potential active sites. Chem. Commun. 2007, 32, 3371-3373. [CrossRef]

6. Fujita, T.; Guan, P.; McKenna, K.; Lang, X.; Hirata, A.; Zhang, L.; Tokunaga, T.; Arai, S.; Yamamoto, Y.; Tanaka, N.; et al. Atomic origins of the high catalytic activity of nanoporous gold. Nat. Mater. 2012, 11, 775-780. [CrossRef] [PubMed]

7. Sil, D.; Gilroy, K.D.; Niaux, A.; Boulesbaa, A.; Neretina, S.; Borguet, E. Seeing Is Believing: Hot Electron Based Gold Nanoplasmonic Optical Hydrogen Sensor. ACS Nano 2014, 8, 7755-7762. [CrossRef] [PubMed]

8. Mukherjee, S.; Zhou, L.; Goodman, A.M.; Large, N.; Ayala-Orozco, C.; Zhang, Y.; Nordlander, P.; Halas, N.J. Hot-electron-induced dissociation of $\mathrm{H}_{2}$ on gold nanoparticles supported on $\mathrm{SiO}_{2}$. J. Am. Chem. Soc. 2014, 136, 64-67. [CrossRef] [PubMed] 
9. Zorić, I.; Zäch, M.; Kasemo, B.; Langhammer, C. Gold, Platinum, and Aluminum Nanodisk Plasmons: Material Independence, Subradiance, and Damping Mechanisms. ACS Nano 2011, 5, 2535-2546. [CrossRef] [PubMed]

10. Lu, C.; Lipson, R.H. Interference lithography: A powerful tool for fabricating periodic structures. Laser Photonics Rev. 2010, 4, 568-580. [CrossRef]

11. Fujitani, T.; Nakamura, I.; Akita, T.; Okumura, M.; Haruta, M. Hydrogen dissociation by gold clusters. Angew. Chem. Int. Ed. 2009, 48, 9515-9518. [CrossRef] [PubMed]

12. Manzoli, M.; Chiorino, A.; Vindigni, F.; Boccuzzi, F. Hydrogen interaction with gold nanoparticles and clusters supported on different oxides: A FTIR study. Catal. Today 2012, 181, 62-67. [CrossRef]

13. Tagliabue, G.; Jermyn, A.S.; Sundararaman, R.; Welch, A.J.; DuChene, J.S.; Pala, R.; Davoyan, A.R.; Narang, P.; Atwater, H.A. Quantifying the role of surface plasmon excitation and hot carrier transport in plasmonic devices. Nat. Commun. 2018, 9, 3394-3402. [CrossRef] [PubMed]

14. Wang, Z.; Du, J.; Zhang, Y.; Han, J.; Huang, S.; Hirata, A.; Chen, M. Free-standing nanoporous gold for direct plasmon enhanced electro-oxidation of alcohol molecules. Nano Energy 2019, 56, 286-293. [CrossRef]

(C) 2019 by the authors. Licensee MDPI, Basel, Switzerland. This article is an open access article distributed under the terms and conditions of the Creative Commons Attribution (CC BY) license (http://creativecommons.org/licenses/by/4.0/). 